\title{
The effectiveness of group problem-solving therapy on women's sexual function and satisfaction after mastectomy surgery
}

\author{
Mahshid Bokaie $^{1}$ (1), Ommolbanin Firouzabadi ${ }^{*}$ (ID) and Azadeh Joulaee ${ }^{3}$ (D)
}

\begin{abstract}
Background: Breast cancer is the second cause of death and the most common cancer in women worldwide, threatening different aspects of individual and mental health, quality of life, sexual function, and sexual satisfaction. This study aimed to determine the effectiveness of group counseling based on a problem-solving solution on women's sexual function and satisfaction after mastectomy surgery.

Methods: The present research was an open pilot study, with a pretest, a post-test, and a follow-up period. Of women referred to the Tehran Breast Cancer Institute, 32 were selected using convenience sampling. The group received eight 90 -min sessions of problem-solving solution counseling. This approach is based on cognitive-behavioral therapy and can improve an individual's ability to cope with stressful life experiences. The data collection tool was FSFI and sexual satisfaction questionnaires, which were filled before the intervention (baseline), immediately after the intervention, and one month later (follow up). Data analysis was performed using SPSS 21 statistical software application at the certainty level of $95 \%(P<0.05)$.
\end{abstract}

Results: The mean FSFI score increased from $18.37 \pm 8.35$ before the intervention to $20.88 \pm 7.67$ immediately after the intervention and $22.95 \pm 5.79$ one month later $(P<0.0001)$. Also, the mean sexual satisfaction score was $65.27 \pm 5.98,68.08 \pm 5.61$, and $70.46 \pm 5.35$ before the intervention, immediately after the intervention, and one month later, respectively $(P<0.05)$. The results also showed that although the two components of sexual function and satisfaction were statistically significant after sexual counseling, this improvement was not clinically progressive. The mean sexual function and satisfaction score was still low after sexual counseling.

Conclusions: It was observed that sexual function and satisfaction were improving among the patients after the intervention. Thus, sexual health counseling sessions are recommended for breast cancer patients.

Keywords: Breast neoplasms, Mastectomy, Sexual dysfunction, Sexual satisfaction, Problem-solving, Women

\section{Background}

Breast cancer constitutes almost one-fourth of all cancers in women [1], and it is the most common cancer among them. One out of eight women is suffering from breast cancer, afflicting almost 1.5 million women each year.

*Correspondence: O-firouzabadi@farabi.tums.ac.ir

2 Shahid Sadoughi University of Medical Sciences, Yazd, Iran

Full list of author information is available at the end of the article
Moreover, women dying from breast cancer stand at the top of the mortality list. The incidence of breast cancer in Iran is reported as 31 per 100,000 women, and the cancer is most prevalent in the $42-49$ age group [2]. Breast cancer is the most common type of cancer (fifth in cancer mortality) in Iranian women [3]. It affects both physical and mental dimensions in an individual [4]. In most societies and cultures, the breast is considered a female organ, and because of its importance in forming the 
female identity [5], reaction to breast cancer can include fear, anxiety, and depression. Some of the new causes of these problems create implicit meanings of this diagnosis in the patient's mind including the likelihood of body deformation, pain, lack of financial and social support, losing female identity and sexual desire, reduced social activity, concern about ambiguous future, recurrence of disease, and death [6].

Iranian women with breast cancer greatly focus on their maternal role, have a great emotional involvement with their children, and effectively cope with their maternal role. Moreover, in the Iranian culture, the wife and the maternal role significantly contribute to women's identity [7].

In addition, Iranian women feel great commitment to fulfill their husbands' sexual needs to protect their families and therefore attach great value to their husbands' patience for their probable sexual problems [8].

Female sexual dysfunction (FSD) is increasingly being identified as a problem worldwide. Women can have problems in various parts of the sexual cycle including desire, arousal, lubrication, orgasm, satisfaction, or pain experience related to sexual activity [9]. Sexual changes are associated with emotional consequences, feeling unattractive or lacking femininity, and concerns about their impact on the partner or relationship [10]. Sexual dysfunction possibly remains more than one year after breast cancer diagnosis. It may be that chemotherapy is the cause of all sexual problems such as reduced sexual desire and mental arousal, vaginal dryness, and dyspareunia [11]. It seems that mastectomy directly impacts the sexual function of women [12].

Sexual satisfaction is a multi-component concept including emotional and physiological aspects of sexual life. Sexual satisfaction is not limited to physical joy, and it includes all positive and negative emotions after sexual intercourse [13]. Providing counseling services for patients reduces their tension so that they consider others' counsels and assistance vital for compatibility with their emotions [14]. Group counseling is a two-sided process in which the counselor and the identical group study problems and approaches. In a group counseling environment, members show a large degree of reaction toward each other, which increases insight acquisition. Counseling programs can improve sexual function and satisfaction [15]. Problem-solving treatment has been used to help people with cancer generate and evaluate various solutions for challenges they face in life [16].

Hummel et al. [17] designed internet-based cognitive behavioral therapy to improve sexual dysfunctions in women treated for breast cancer.

Educating the problem-solving issue mentions a cognitive-behavioral process that provides a variety of alternative and potential responses for controlling the problematic conditions and increases the possibility of choosing the best and most effective alternative responses. The advantage of the problem-solving method is that we can use it for individual and/or group treatments. This approach is based on cognitive-behavioral therapy and could improve an individual's ability to cope with stressful life experiences [18]. This type of treatment will initiate active cooperation between the patient and the therapist [19]. Problem-solving treatment is a brief, structured psychological intervention. The treatment shares with other cognitive-behavioral treatments a focus on here and now rather than dwelling on past experiences and regrets. The treatment involves active collaboration between the patient and the therapist, with the patient taking an increasingly active role in planning treatment and implementing activities between treatment sessions [20].

\section{Aim of the study}

This study aimed to determine the effectiveness of group counseling based on a problem-solving solution on women's sexual function and satisfaction after mastectomy surgery.

\section{Methods \\ Design and setting}

The present research was a semi-experimental study, with a pretest, a post-test, and a one-month follow-up period. Written informed consent was obtained from all the participants. The patients were contacted based on the list available at the institute and according to the admission criteria. The samples were selected based on the inclusion criteria by reviewing the existing files. Breast cancer patients can choose this institution for treatment and use its services. Of 40 patients referred to the Tehran Breast Cancer Institute between Aug and Dec 2020, 32 were invited to participate in the study according to the inclusion and exclusion criteria. The sample size formula was $\left(N=\frac{2 *\left(Z_{\left(1-\frac{\alpha}{2}\right)}+Z_{(1-\beta)}\right)^{2} * p * q}{\left(p_{0}-p_{1}\right)^{2}}\right.$ where $\boldsymbol{p}=\frac{\boldsymbol{p}_{0}-\boldsymbol{p}_{1}}{2}, \boldsymbol{q}=1-\boldsymbol{p}$, with \%95 confidence interval, \%5 alpha, and \%15 chance of falling). The students were assured that the study results were confidential and would be published without names. They could leave the research at any stage.

\section{Eligibility criteria}

The inclusion criteria were as follows: cancer stages between I and III, 1-5 years passing from breast surgery, Iranian nationality, residence in Tehran, 30-59 years of age, ability to read and write, mastectomy, being married, 
having a single partner, not participating in any other consulting class, and finished chemotherapy.

The exclusion criteria were lumpectomy surgery, cancer recurrence, history of significant physical and mental illnesses such as schizophrenia and major depression, and drug abuse.

\section{Instruments}

The research instruments included a demographic characteristics questionnaire, the female sexual function index (FSFI) questionnaire [21], and the Larson sexual satisfaction questionnaire [22]. The demographic characteristics questionnaire included age, education, marital status, contraceptive method, breast cancer grading, type of surgery, chemotherapy, and radiotherapy records.

The female sexual function index (FSFI) includes 19 questions, each with four answer options. This standard questionnaire measures six dimensions of sexual function (sexual desire (two items), sexual arousal (four items), lubrication (four items), orgasm (three items), sexual satisfaction (three items), and pain (three items) over the recent four weeks. Measures were taken as per the questionnaire to determine each person's score in each section and determine the overall score. The lowest score of 2 is the maximum of 36 . The cutting score for determining sexual disorders is 26 or less. The total point obtained is calculated in different areas. The total score is obtained by adding up six sections Brown, 2000). The questionnaire was validated by Fakhri et al. The general test-retest reliability coefficients were acceptable for each domain of the questionnaire (from 0.73 to 0.86 ) and the internal consistencies (from 0.72 to 0.90 ) [24].

The sexual satisfaction questionnaire is the Iranian version of the sexual satisfaction questionnaire consisting of 255 -answer questions. The items in the questionnaire are scored based on a Likert scale from one to five, with "never" receiving 1 , "rarely" receiving 2 , "sometimes" receiving 3 , "most of the times" receiving 4 , and "always" receiving 5. A total score of $25-75$ is equal to low sexual satisfaction, 76-100 to medium sexual satisfaction, and 101-125 to high sexual satisfaction. Reliability was determined using Cronbach's alpha coefficient ( 0.8 for positive question and 0.77 for negative one) and intra-class correlation coefficients $(\mathrm{ICC}=0.8)(23,24)$.

\section{Interventions}

This study was accomplished in fall and winter 2020 . After obtaining informed consent, the participants provided their phone numbers and addresses to participate in the study. The time, date, and place of the counseling sessions were announced by phone. A counseling program based on a problem-solving approach, including eight 90-min counseling sessions per week, was designed for the participants based on a review of texts and the research team's opinions. The participants formed four groups, each consisting of eight individuals in a suitable location in the breast cancer institute. A summary of the contents of the sessions is outlined in Table 1. The questionnaires were completed by the participants before the intervention (baseline), immediately after the intervention, and one month later (follow up).

Table 1 Subjects of discussions in each counseling session

\begin{tabular}{|c|c|}
\hline Sessions & Subjects of discussion \\
\hline One & $\begin{array}{l}\text { The introduction, anatomy, and physiology of the reproductive system, the sexual cycle, the female sexual organ and erogenous zones and } \\
\text { factors affecting them, explanation on how to fill in the questionnaire, and presenting home assignments }\end{array}$ \\
\hline Two & $\begin{array}{l}\text { The importance of sexual relation and the lack of concentration on penetration in all sexual relations of the couple, speaking about cancer } \\
\text { and its effects, treatments, and complications, presenting homework, and explanation on how couple get along with this problem }\end{array}$ \\
\hline Three & $\begin{array}{l}\text { Training problem-solving skills, confronting with stress, techniques of relaxation and establishing effective communication, exchange of } \\
\text { views regarding alternative solutions for improving the couple's relation in breast cancer, giving home assignments }\end{array}$ \\
\hline Four & $\begin{array}{l}\text { The review of sexual schemas and women's concerns and their control, review of their sexual expectations, training sexual skills, sensational } \\
\text { exercises, necessary readiness for relation, explanation regarding home assignments such as common massage, and giving home assign- } \\
\text { ments }\end{array}$ \\
\hline Five & $\begin{array}{l}\text { Free discussion regarding issues and problems, defining exact conformation of the issue, determining a range of possible solutions, analyz- } \\
\text { ing solutions, problems created following the execution of individual solutions, weaknesses, and strengths of individual solutions, anticipat- } \\
\text { ing consequences, developing and strengthening communication skills, managing negative excitement (negative anger and excitement), } \\
\text { training relaxation, responding to questions, and giving home assignments }\end{array}$ \\
\hline Six & $\begin{array}{l}\text { Providing suitable solutions against complications caused by cancer in sexual matters, changes in appearance, improving communication } \\
\text { techniques, reducing worries through counseling, providing introduction to the Kegel exercise and other effective factors in strengthening } \\
\text { the sexual function (nutrition, concentration on moments of enjoying it), responding to questions, and giving home assignments }\end{array}$ \\
\hline Seven & The review of previous sessions and implementing a solution for real cases set forth \\
\hline Eight & $\begin{array}{l}\text { The presence of spouses and study of their sexual expectations, talking about sexual and mental differences of men and women in each } \\
\text { stage of the sexual cycle, sexual interactions, and sexual response and solutions to improve sexual satisfaction, explaining on how to fill in } \\
\text { the questionnaire }\end{array}$ \\
\hline
\end{tabular}




\section{Ethical considerations}

The study was performed under the Declaration of Helsinki and approved by the Ethics Committee of the Research Deputy of the Shahid Sadoughi University of Medical Sciences (code: IR.SSU.MEDICINE. REC.1397.176).

After explaining the study's aims for the participants, written informed consent was obtained from all of the participants. Confidentiality was ensured.

\section{Data analysis}

The data were analyzed using descriptive statistics and inferential statistics via SPSS 21 software (SPSS, Inc., Chicago, IL, USA). A significant value was considered less than 0.05. Since the distributions of the studied variables were normal, parametric statistical tests, such as variance analysis used, repeated measures, and the Bonferroni post hoc test, were performed.

The training of the five problem-solving skills began based on the problem-solving steps of Dezorella and Goldfried. The steps included defining and planning the problem, analyzing the problem, determining real goals, producing a solution, deciding and choosing the best solution, predicting possible consequences of each action, paying attention to the usefulness of these consequences, implementing the selected solution, and reviewing and evaluating the steps of problem-solving skills.

Sample assignments:

- A list of symptoms and complications of treatment was prepared and shared in the next consultation

- Preparing a list of ways to deal with negative thoughts and strengthen positive thoughts, reviewing solutions

- Practicing problem-solving skills (reducing intimacy and fear of having sex) and practicing strategies and suggestions in the following counseling session

- Practicing anger management strategies, reducing negative emotions, practicing active listening, and sharing results in the following counseling session

- Dedicating time and space to oneself, practicing relaxation skills, practicing sensory exercises, breathing training, practicing joint massage, and Kegel exercising and sharing it with one's spouse

Due to the extent of the problem in counseling sessions, the problem-solving and cognitive-behavioral methods became a component.

\section{Results}

This study was performed on 32 women after mastectomy referred to the Tehran Breast Cancer Institute. The average age of the women was $39.81 \pm 7.54$, the average spouse's age was $43.81 \pm 8.67$, the marriage duration average was $14.9 \pm 81.59$, the cancer affliction period was $4.69 \pm 3.08$, and the post-treatment interval was $3.47 \pm 3.12$. All the participants experienced chemotherapy, and 27 (84.4\%) of them also experienced radiotherapy. Some of the demographic characteristics are mentioned in Table 2.

The mean sexual satisfaction score was $65.27 \pm 5.98$, $68.08 \pm 5.61$, and $70.46 \pm 5.35$ before the intervention, immediately after the intervention, and one month later, respectively. Table 3 shows the statistical test of a significant difference in the three periods $(P<0.05)$.

The mean FSFI score increased from $18.37 \pm 8.35$ before the intervention to $20.88 \pm 7.67$ immediately after the intervention () and $22.95 \pm 5.79$ one month later (follow up) $(P<0.0001)$. The mean score of the sixfold dimensions of sexual function is shown in Table 4.

Table 2 The descriptive characteristics of the women after mastectomy surgery

\begin{tabular}{|c|c|c|}
\hline Variable & Number & Percentage \\
\hline \multicolumn{3}{|l|}{ Women education } \\
\hline Under diploma & 6 & 18.8 \\
\hline Diploma & 11 & 34.4 \\
\hline University degrees & 15 & 46.8 \\
\hline \multicolumn{3}{|l|}{ Spouse education } \\
\hline Under diploma & 6 & 18.8 \\
\hline Diploma & 11 & 34.4 \\
\hline University degrees & 15 & 46.8 \\
\hline \multicolumn{3}{|l|}{ Women's occupation } \\
\hline Housewife & 20 & 62.5 \\
\hline Employed & 12 & 37.5 \\
\hline \multicolumn{3}{|l|}{ Spouse's occupation } \\
\hline Employee & 10 & 31.2 \\
\hline Self-employed & 3 & 9.4 \\
\hline Laborer & 19 & 59.4 \\
\hline \multicolumn{3}{|l|}{ Contraception method } \\
\hline Hormonal & 3 & 9.4 \\
\hline IUD & 7 & 21.8 \\
\hline Withdrawal & 18 & 56.3 \\
\hline $\mathrm{TL}$ & 4 & 12.5 \\
\hline \multicolumn{3}{|l|}{ Cancer grading } \\
\hline । & 6 & 18.8 \\
\hline$\|$ & 21 & 65.6 \\
\hline III & 4 & 12.5 \\
\hline IV & 1 & 3.1 \\
\hline
\end{tabular}


Table 3 The mean difference of sexual satisfaction in three periods

\begin{tabular}{|c|c|c|c|c|}
\hline Time & Mean $\pm S D$ & Time & Mean $\pm S D$ & $P^{*}$ \\
\hline Before intervention (baseline) & $65.27 \pm 5.98$ & Immediately after intervention & $68.08 \pm 5.61$ & 0.02 \\
\hline Immediately after intervention & $68.08 \pm 5.61$ & One month later (follow-up) & $70.46 \pm 5.35$ & 0.03 \\
\hline One month later (follow-up) & $70.46 \pm 5.35$ & before Intervention (baseline) & $65.27 \pm 5.98$ & 0.008 \\
\hline
\end{tabular}

${ }^{*} P<0.05$ shows the statistical test of a significant difference in the three periods

Table 4 Comparison of the mean total score of sexual function and its dimensions in three periods

\begin{tabular}{|c|c|c|c|c|c|}
\hline Dimensions of FSFI & $\begin{array}{l}\text { Before intervention } \\
\text { (baseline) } \\
\text { (Mean } \pm \text { SD) }\end{array}$ & $\begin{array}{l}\text { Immediately after } \\
\text { intervention } \\
(\text { Mean } \pm \text { SD) }\end{array}$ & $\begin{array}{l}\text { One month after intervention } \\
\text { (follow-up) } \\
\text { (Mean } \pm \text { SD) }\end{array}$ & $F$ & $P^{*}$ \\
\hline Total score & $18.37 \pm 8.35$ & $20.88 \pm 7.67$ & $22.95 \pm 5.79$ & 20.53 & $<0.001$ \\
\hline Sexual desire & $2.98 \pm 0.67$ & $2.99 \pm 0.68$ & $3.11 \pm 0.71$ & 15.96 & $<0.001$ \\
\hline Sexual arousal & $2.98 \pm 1.30$ & $3.22 \pm 1.04$ & $4.43 \pm 0.98$ & 6.98 & $<0.003$ \\
\hline Lubrication & $2.91 \pm 1.47$ & $3.36 \pm 1.32$ & $4.02 \pm 0.99$ & 24.02 & $<0.001$ \\
\hline Orgasm & $3.24 \pm 1.78$ & $3.81 \pm 1.36$ & $4.08 \pm 0.96$ & 10.39 & $<0.001$ \\
\hline Satisfaction & $3.39 \pm 1.67$ & $3.90 \pm 1.54$ & $4.07 \pm 1.02$ & 11.63 & $<0.001$ \\
\hline Pain & $3.18 \pm 2.08$ & $3.60 \pm 1.73$ & $3.24 \pm 1.14$ & 10.51 & $<0.001$ \\
\hline
\end{tabular}

${ }^{*} P<0.05$ shows the statistical test of a significant difference in the three periods

Table 5 Comparison of the mean total score of sexual function in two periods using the Bonferroni test

\begin{tabular}{lllll}
\hline Time & Mean \pm SD & Time & Mean \pm SD & $\boldsymbol{P}^{*}$ \\
\hline Before intervention (baseline) & $18.37 \pm 8.35$ & Immediately after intervention & $20.88 \pm 7.67$ & $<0.001$ \\
Immediately after intervention & $20.88 \pm 7.67$ & One month later (follow-up) & $22.95 \pm 5.79$ & $<0.001$ \\
One month Later (follow-up) & $22.95 \pm 5.79$ & Before intervention (baseline) & $18.37 \pm 8.35$ & $<0.001$ \\
\hline
\end{tabular}

${ }^{*} P<0.05$ shows the statistical test of a significant difference in the three periods

Table 5 compares the mean total score of sexual function in the two periods using the Bonferroni test.

\section{Discussion}

This study aimed to determine the effectiveness of group counseling based on problem-solving solutions on women's sexual function and satisfaction after mastectomy surgery.

Other findings of the present study indicated that group counseling based on problem-solving solutions had no clinical effect on women's sexual function and satisfaction after mastectomy surgery.

Numerous studies have been conducted on psychological counseling issues in women with breast cancer [27, 28]. However, few studies have been performed on the effect of sexual health counseling on women with breast cancer. Studies carried out in different countries have repeatedly stated that women feel that their sexual function is affected by mastectomy.
Molavi et al. [29] evaluated couples' sexual function and satisfaction after mastectomy surgery. In this study, the mean FSFI score was estimated in mastectomy patients, and most of them reported moderate sexual satisfaction.

Many studies addressed the sexual problems of women with breast cancer. In a study conducted by Kedde et al. [30], young women undergoing breast cancer treatment had a more negative experience about sexuality and were less sexually active [30]. Zahedian et al. [31] showed that group meta-cognitive therapy improved depression in women with breast cancer.

Hamzehgardeshi et al. [32] showed the effect of midwifery-based counseling support programs on the body image of breast cancer women survivors.

The mean FSFI score of mastectomy patients in their study is in line with that in our research. However, the mean sexual satisfaction score in our study is lower than that in their study. Reese et al. [33] showed that sexual concerns for breast cancer survivors were not mostly removed, and evidence-based interventions 
were necessary, particularly in cases that the survivors had partners [33]. This study confirms the participation of couples in the intervention that we also proposed.

Fatehi et al. [34] showed that the psychosexual program effectively improved sexual function and quality of sexual life among Iranian breast cancer survivors.

A recent study is observed to be in line with our study regarding women's sexual function. In this study, although FSFI was statistically significant after the intervention, sexual function did not overtake from the cut off point. Sexual satisfaction in our study statistically significantly improved from $65.27 \pm 5.98$ to $68.08 \pm 5.61$. However, Fatehi et al. reported no change in the statistical and clinical dimensions.

Combining problem-solving with another method and continuing group counseling sessions seem to be highly effective.

We were not able to include couples in the study due to limitations. Studies on interventions performed on couples are highly effective. In our opinion, if, after the diagnosis of cancer, step-by-step counseling sessions are performed simultaneously with the treatment steps and according to the individual's needs, the intervention will be highly effective and efficient.

\section{Study limitations}

None of the participants wanted to be in the control group. Therefore, this study was not a randomized clinical trial. Also, many women after mastectomy surgery were not members of the Tehran Breast Cancer Center. Moreover, due to cultural reasons, meetings were not held for the spouses, and couple therapy is recommended for these patients. The short-term follow-up period (one month) was another limitation of this study.

\section{Conclusion}

The present study results showed that although the two components of sexual function and satisfaction were statistically significant after sexual counseling, this improvement was not clinically progressive. The mean sexual function and satisfaction score was still low after sexual counseling. As sexual function and satisfaction improve, continuing sexual health counseling sessions for breast cancer patients is recommended.

\section{Abbreviations}

FSFI: The Female Sexual Function Index; FSD: Female Sexual Dysfunction.

\section{Acknowledgements}

This paper was extracted from a Master's thesis in counseling in midwifery approved by the Shahid Sadoughi University of Medical Sciences. We would like to express our appreciation to all the personnel of the Tehran Breast Cancer Institute for their cooperation in the execution of this research and the personnel of the Shahid Sadoughi University of Medical Sciences.

\section{Authors' contributions}

O.F: Conception and design, provision of study materials of patients, data collection and assembly, data analysis and interpretation, article writing, reviewing, and editing. M.B and A.J: Conception and design, reviewing, and editing. All the authors read and approved the final manuscript.

Funding

Not applicable.

\section{Availability of data and materials}

The datasets used and analyzed during the present study are available from the corresponding author on reasonable request.

\section{Declarations}

\section{Ethics approval and consent to participate}

The study was approved by the Ethics Committee of the Research Deputy of the Shahid Sadoughi University of Medical Sciences (code: IR.SSU.MEDICINE. REC.1397.176). Based on the list available at the institute, first, the research objectives and the reason for conducting the research were explained to the participants via text messages or phone calls. After calling the participants and obtaining more information, they announced their consent or dissatisfaction to enter the study. It was emphasized that refusing to participate in the study would not interfere with providing regular care for the patients. After explaining the study's aims to the participants, written informed consent was obtained from them, and their confidentiality was ensured.

\section{Consent for publication}

Not applicable.

\section{Competing interests}

The authors declare that they have no competing interests.

\section{Author details}

${ }^{1}$ Research Center for Nursing and Midwifery Care, Shahid Sadoughi University of Medical Sciences, Yazd, Iran. ${ }^{2}$ Shahid Sadoughi University of Medical Sciences, Yazd, Iran. ${ }^{3}$ Special Breast Unit, Mahdieh University Women's Hospital, Shahid Beheshti University of Medical Sciences, Tehran, Iran.

Received: 4 March 2021 Accepted: 15 February 2022

Published online: 23 February 2022

References

1. Heravi KM, Pourdehghan M, Jadid MM, Forotan SK, Aein F. Effect of group counseling on sexual health of patients with breast cancer. Sci J Forensic Med. 2006;11:201-6.

2. Diagnosis screening. https://www.who.int/cancer/prevention/diagn osis-screening.

3. Rahimzadeh M, Pourhoseingholi MA, Kavehie B. Survival rates for breast cancer in Iranian patients: a meta-analysis. Asian Pac J Cancer Prev. 2016;17:2223-7.

4. Shayan A, Khalili A, Rahnavardi M, Masoumi SZ. The relationship between sexual function and mental health of women with breast cancer. Avicenna J Nurs Midwifery Care (Sci J Hamadan Nurs Midwifery Fac). 2017;24:221-7.

5. Mofrad SA, Fernandez R, Lord H, Alananzeh I. The impact of mastectomy on Iranian women sexuality and body image: a systematic review of qualitative studies. Support Care Cancer. 2021;29:5571-80.

6. Black JM, Hawks JH. Medical-surgical nursing. St. Louis: Elsevier; 2005.

7. Vaziri S, Lotfi Kashani F, Akbari ME, Ghorbani AY. Comparing the motherhood and spouse role in women with breast cancer and healthy women. Iran Q J Breast Dis. 2014;7:76-83.

8. Foroutan SK, Jadid MM. The prevalence of sexual dysfunction among divorce requested. Daneshvar Med Basic Clin Res J. 2008;15:39-44.

9. Safdar F, Eng CL, Wai KL, Tey WS, Ang SB. Prevalence of female sexual dysfunction in allied health workers: a cross-sectional pilot study in a tertiary hospital in Singapore. BMC Womens Health. 2019;19:1-7. 
10. Ussher JM, Perz J, Gilbert E. Information needs associated with changes to sexual well-being after breast cancer. J Adv Nurs. 2013;69:327-37.

11. Panjari M, Bell RJ, Davis SR. Sexual function after breast cancer. J Sex Med. 2011:8:294-302.

12. Fahami F, Savabi M, Mohamadirizi S. Relationship of sexual dysfunction and its associated factors in women with genital and breast cancers. Iran J Nurs Midwifery Res. 2015;20:516.

13. Mofid V, Ahmadi A, Etemadi O. The comparison of Cognitive-Behavioral counseling and solution-oriented counseling on women's sexual satisfaction in Isfahan. Sociol Women (J Woman Soc). 2014;5:67-83.

14. Ramezani T. Requiring depression counseling for women with breast cancer. Andishe va Raftar. 2001;6:70-7.

15. Navabinezhad SH. Guidance and group counseling. Tehran Organ Study Codif Soc Sci Books. 2010.

16. Nezu AM, Nezu CM, Friedman SH, Faddis S, Houts PS. Helping cancer patients cope: a problem-solving approach. Washington, DC: American Psychological Association; 1998.

17. Hummel SB, van Lankveld JJDM, Oldenburg HSA, Hahn DEE, Broomans E, Aaronson NK. Internet-based cognitive behavioral therapy for sexual dysfunctions in women treated for breast cancer: design of a multicenter, randomized controlled trial. BMC Cancer. 2015;15:1-12.

18. Gellis ZD, Kenaley B. Problem-solving therapy for depression in adults: a systematic review. Res Soc Work Pract. 2008;18:117-31.

19. Mynors-Wallis L. Problem-solving treatment in general psychiatric practice. Adv Psychiatr Treat. 2001;7:417-25.

20. Chen C-P, Huang K-G, Wan G-H, Tu L-Y, Lee J-T. Sexual satisfaction and related factors in women previously treated for gynecological cancer. Hu Li Za Zhi. 2013;60:61.

21. Mohammady K, Heidari M, Faghih ZS. Validity of the Persian version of Female Sexual Function Index-FSFI scale as the Female Sexual Function Index. J Payesh. 2008:7:269-78.

22. Shams MZ, Shahsiah M, Mohebi S, Tabaraee Y. The effect of marital counseling on sexual satisfaction of couples in Shiraz city. Health Syst Res. 2010;6:417-24

23. Rosen C, Brown J, Heiman S, Leiblum C, Meston R, Shabsigh D, Ferguson R, D'Agostino R. The Female Sexual Function Index (FSFI): a multidimensional self-report instrument for the assessment of female sexual function. J Sex Marital Ther. 2000;26:191-208.

24. Fakhri A, Pakpour AH, Burri A, Morshedi H, Zeidi IM. The Female Sexual Function Index: translation and validation of an Iranian version. J Sex Med. 2012;9:514-23.

25. Bahrami N, Sharif Nia H, Soliemani MA, Haghdoost AA, et al. Validity and reliability of the persian version of Larson sexual satisfaction questionnaire in couples. J Kerman Univ Med Sci. 2016;23:344-56.

26. Gheshlaghi F, Dorvashi G, Aran F, Shafiei F, Najafabadi GM. The study of sexual satisfaction in Iranian women applying for divorce. Int J Fertil Steril. 2014;8:281.

27. Li L, Yang Y, He J, Yi J, Wang Y, Zhang J, et al. Emotional suppression and depressive symptoms in women newly diagnosed with early breast cancer. BMC Womens Health. 2015;15:1-8.

28. Ramos C, Leal I, Tedeschi RG. Protocol for the psychotherapeutic group intervention for facilitating posttraumatic growth in nonmetastatic breast cancer patients. BMC Womens Health. 2016;16:1-9.

29. Molavi A, Hekmat K, Afshari P, Hoseini M. Evaluation of couples' sexual function and satisfaction after mastectomy. Iran J Obstet Gynecol Infertil. 2015;17:17-24.

30. Kedde H, de Wiel HBM, Schultz WCMW, Wijsen C. Subjective sexual wellbeing and sexual behavior in young women with breast cancer. Support Care Cancer. 2013;21:1993-2005.

31. Zahedian E, Bahreini M, Ghasemi N, Mirzaei K. Group meta-cognitive therapy and depression in women with breast cancer: a randomized controlled trial. BMC Womens Health. 2021;21:1-9.

32. Hamzehgardeshi Z, Moosazadeh M, Elyasi F, Janbabai G, Rezaei M, Yeganeh Z, et al. Effect of midwifery-based counseling support program on body image of breast cancer women survivors. Asian Pac J Cancer Prev APJCP. 2017;18:1293.

33. Reese JB, Zimmaro LA, Lepore SJ, Sorice KA, Handorf E, Daly MB, et al. Evaluating a couple-based intervention addressing sexual concerns for breast cancer survivors: study protocol for a randomized controlled trial. Trials. 2020;21:1-13
34. Fatehi S, Maasoumi R, Atashsokhan G, Hamidzadeh A, Janbabaei G, Mirrezaie SM. The effects of psychosexual counseling on sexual quality of life and function in Iranian breast cancer survivors: a randomized controlled trial. Breast Cancer Res Treat. 2019;175:171-9.

\section{Publisher's Note}

Springer Nature remains neutral with regard to jurisdictional claims in published maps and institutional affiliations.
Ready to submit your research? Choose BMC and benefit from:

- fast, convenient online submission

- thorough peer review by experienced researchers in your field

- rapid publication on acceptance

- support for research data, including large and complex data types

- gold Open Access which fosters wider collaboration and increased citations

- maximum visibility for your research: over $100 \mathrm{M}$ website views per year

At $\mathrm{BMC}$, research is always in progress.

Learn more biomedcentral.com/submissions 